\title{
The validity of surgical simulation
}

\author{
Riaz A. Agha, MBBS, MSc* \\ Alexander J. Fowler ${ }^{\dagger}$
}

From the *Department of Plastic Surgery, Stoke Mandeville Hospital, Stoke Mandeville, Ayelsbury, Bucks, UK, and †Barts and the London School of Medicine and Dentistry, Queen Mary, University of London, UK

Accepted for publication

Dec. 4, 2013

\author{
Correspondence to: \\ A.J. Fowler \\ London School of Medicine \& Dentistry \\ Garrodd Building \\ Turner Street, Whitechapel \\ London, UK \\ E1 2AD \\ alexjfowler@gmail.com
}

DOI: $10.1503 /$ cjs.032013

\section{- Summary}

Simulation is playing an increasingly important role in training surgeons. As hours between registrar and consultant grades have decreased, trainees are required to train smarter. While the majority of simulation is limited, advances in computing and design are enabling ever more realistic, varied simulation.

imulation is an important tool in the training of juniors, but work is required to expand this training to a wider variety of surgical techniques, not only laparoscopic ones. The very first surgical simulators were leaf and clay models used in India in $600 \mathrm{BC}$ to simulate a forehead nasal flap reconstruction. ${ }^{1}$ Since then, simulation has become a highly refined training format that is used in number of high-risk industries. It has become a key tool in the education of clinicians at all levels in a wide selection of specialties and is an important component in recent drives to improve patient safety.

A large proportion of the methodological and technological development in simulation has been in the aviation industry, where pilots have long been trained to fly before stepping into an aircraft. ${ }^{2}$ In the United Kingdom, the combination of many registrar grades into the single grade of "Specialist Registrar" (known as Calmanisation) and the European Working Time Directive have reduced the period available for training. As such, the working hours between becoming a senior house officer and a consultant have estimated to have reduced by a factor of $5 .^{3}$ Simulation has evolved as an effective training technique alongside this changing environment for the training of surgeons - namely the reduction in hours available for training.

This drastic change in training time and practices necessitated a paradigm shift in the model of surgical education. There has been a move away from the apprenticeship model in which expertise was acquired through experience, to a more standardized, objective and competency-based approach that requires a more proactive attitude to training. Simulation has become a key part of providing this objective training and assessment, allowing mistakes to be made in a safe environment and to develop further attributes, such as understanding human factors, that exist outside the realm of pure technical ability.

Much work has been carried out looking into the use of laparoscopic simulators. Seymour and colleagues ${ }^{4}$ randomized 16 surgical trainees to either a laparoscopic simulator (MIST-VR; Virtalis) training group or a control group trained traditionally. Participants then performed a cholecystectomy in an operating theatre, and the procedures were recorded for assessment. Participants in the simulator group dissected the gallbladder $29 \%$ faster and were 5 times less likely to make errors than those in the control group. These findings were supported in a similar investigation undertaken by Grantcharov and colleagues ${ }^{5}$ involving laparoscopic novices. The MIST-VR group performed significantly faster than the control group, with better economy of movement and error scores. A recent 
systematic review of laparoscopic surgery simulation encompassing 219 studies and 7138 trainees concluded that "simulation-based laparoscopic training of health professionals [has] large benefits when compared with no intervention and is moderately more effective than nonsimulation instruction." 6

Work by Kneebone and colleagues ${ }^{7}$ has taken the concept of simulation a step further: "simulated patients" force trainees to interact with real people while performing procedures. This technique has been extended to laparoscopic surgery, where tactile feedback allows trainees to undertake the operation with a number of anatomic variants and get used to the feeling of handling different tissues. Alongside these technically useful features, authenticity is enhanced by giving the model patient head and feet, artificial skin and a theatre team, including all those normally present for such an operation. The quality of simulator used may impact the outcomes for patients - simulators without haptics can lead to distortions of pulling and pushing forces required. ${ }^{8}$ Much work still remains to be done on transferring teamwork and leadership skills as well as human factors from the simulation suite to the operating room.

Growing evidence suggests that skills gained within simulated environments transition well into the real clinical setting. A recent review found good skill transfer in pediatric emergency situations, tracheal intubation and central venous catheter insertion, with reported decreases in complications and infections. Zendejas and colleagues ${ }^{9}$ investigated laparoscopic inguinal hernia repair in a randomized controlled trial ${ }^{10}$ and subsequently found decreased procedure duration and complications. Stefanidis and colleagues ${ }^{11}$ found that $71 \%$ of novices trained to proficiency on a simulator retained their skills in the operating theatre.

The vast majority of work pertaining to skills translation has been undertaken in laparoscopic surgical tech- niques. Future research should examine the wider aspects of surgery. Simulation should be part of the learning experience but cannot replace the requisite clinical hard "graft" and experience a trainee surgeon needs on the "shop floor," supported by good trainers and mentors.

Competing interests: None declared.

\section{References}

1. Limberg AA. The Planning of local plastic operations on the body surface: theory and practice. Lexington (MA): DC Health and Company; 1984.

2. Enochsson L, Isaksson B, Tour R, et al. Visuospatial skills and computer game experience influence the performance of virtual endoscopy. $\mathcal{F}$ Gastrointest Surg 2004;8:876-82.

3. Chikwe J, de Souza AC, Pepper JR. No time to train the surgeons. BM7 2004;328:418-9.

4. Seymour NE, Gallagher AG, Roman SA, et al. Virtual reality training improves operating room performance. Ann Surg 2002;236:458-63.

5. Grantcharov TP, Kristiansen VB, Bendix J, et al. Randomized clinical trial of virtual reality simulation for laparoscopic skills training. $\mathrm{Br} \mathcal{F}$ Surg 2004;91:146-50.

6. Zendejas B, Brydges R, Hamstra SJ, et al. State of the evidence on simulation-based training for laparoscopic surgery: a systematic review. Ann Surg 2013;257:586-93.

7. Kneebone R, Nestel D, Wetzel C, et al. The human face of simulation: patient-focused simulation training. Acad Med 2006;81:919-24.

8. Chmarra MK, Dankelman J, van den Dobbelsteen JJ, et al. Force feedback and basic laparoscopic skills. Surg Endosc 2008;22:2140-8.

9. Zendejas B, Cook DA, Bingener J, et al. Simulation-based mastery learning improves patient outcomes in laparoscopic inguinal hernia repair: a randomized controlled trial. Ann Surg 2011;254:502-9.

10. Griswold S, Ponnuru S, Nishisaki A, et al. The emerging role of simulation education to achieve patient safety: translating deliberate practice and debriefing to save lives. Pediatr Clin North Am 2012; 59:1329-40.

11. Stefanidis D, Yonce TC, Korndorffer JR Jr, et al. Does the incorporation of motion metrics into the existing FLS metrics lead to improved skill acquisition on simulators? A single blinded, randomized controlled trial. Ann Surg 2013;258:46-52. 